\title{
Braking Control for Improving Ride Comfort
}

\author{
Jonghyup Lee ${ }^{1}$, Seibum Choi $^{1}$ \\ ${ }^{1,1 a}$ KAIST, Department of Mechanical Engineering, 34141 Daejeon, Republic of Korea
}

\begin{abstract}
While many vehicle control systems focus on vehicle safety and vehicle performance at high speeds, most driving conditions are very low risk situations. In such a driving situation, the ride comfort of the vehicle is the most important performance index of the vehicle. Electro mechanical brake (EMB) and other brake-by-wire (BBW) systems have been actively researched. As a result, braking actuators in vehicles are more freely controllable, and research on improving ride comfort is also possible. In this study, we develop a control algorithm that dramatically improves ride comfort in low risk braking situations. A method for minimizing the inconvenience of a passenger due to a suddenly changing acceleration at the moment when the vehicle is stopped is presented. For this purpose, an acceleration trajectory is generated that minimizes the discomfort index defined by the change in acceleration, jerk. A controller is also designed to track this trajectory. The algorithm that updates the trajectory is designed considering the error due to the phase lag occurring in the controller and the plant. In order to verify the performance of this controller, simulation verification is completed using a car simulator, Carsim. As a result, it is confirmed that the ride comfort is dramatically improved.
\end{abstract}

\section{Introduction}

Most of the control researches on braking and turning of the vehicle are carried out to improve the safety or the performance of the vehicle in a dangerous situation. However, most of the driving situation is a non dangerous driving situation which does not require the extreme performance of the vehicle. As the autonomous driving researches actively, it is expected that the performance need for the extreme situation of the vehicle is further reduced. For such non- dangerous driving situations, the ride quality of the vehicle is the most important performance indicator. In fact, studies on the relief of passengers' ride comfort have been actively conducted for vehicles with a significantly lower probability of accidents such as wheelchair, elevator [1],[2]. However, in the case of the conventional automobile, the autonomous control cannot be performed due to the hardware structure which depends on the driver input. As the brake by wire (BBW) system such as electro mechanical brake system (EMB) and electro wedge brake system (EWB) is developed in the recent vehicle system, the vehicle can freely control the behavior of the vehicle itself in addition to the cylinder input of the driver. This allows the control system to get rid of the constraints on multiple control situations that depend only on the existing operator input, so that braking control for ride comfort is also possible in the vehicle.

The uncomfortable ride is caused by amount of acceleration and jerk, which is the amount of change in acceleration. Because of the acceleration of the vehicle, the passenger receives the inertia force and the body receives the force. The greater the magnitude of the force or the greater the change in force, the more the passenger feels discomfort. Therefore, in this study, control is performed for the longitudinal braking situation to increase the ride comfort. Define the discomfort index for the ride comfort and create a desired acceleration trajectory that minimizes the discomfort index. In addition, an acceleration controller capable of keeping up with this is designed to perform braking control for ride comfort.

The composition of this paper is as follows. A detailed description of the control target in this study is presented in section 2. Controller design to meet the control target is done in section 3 . Then, the simulation verification and control results via the Carsim are shown in section 4. Finally, conclusions are given in Section 5.

\section{Control target}

The moment when a passenger feels uncomfortable during braking is roughly classified into three types. The first is the inconvenience that the body suddenly slips forward when braking starts, the second is the inconvenience of enduring the inertia force generated in front of the braking system, and the inconvenience that the body suddenly leans back when the vehicle finally stops. This inconvenience is caused by the acceleration that necessarily occurs for the vehicle to stop, and it is determined by the input of the driver for the deceleration capability which is entirely determined by the driver. In 
other words, if the acceleration and the jerk are reduced in size to improve the ride comfort, the ride quality is improved but the possibility of an accident is increased due to the increase of the braking distance. In particular, since the starting point of braking and the amount of deceleration during braking greatly influence the braking distance, it is not suitable as a target of braking control for ride comfort. However, in the case of jerk which occurs at the moment when the vehicle is stopped as shown in Fig. 1, since the control is performed at a low speed, the braking distance is not greatly affected and the ride quality can be improved.

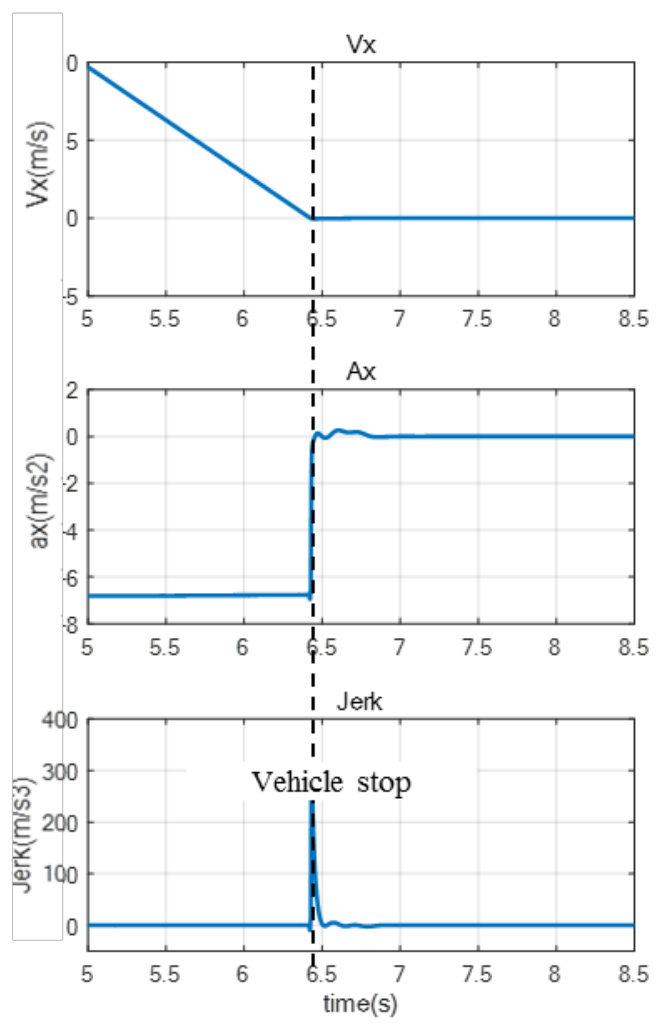

Figure 1. Vehicle speed, acceleration and jerk at vehicle stop.

The principle of the jerk that occurs when the vehicle stops is as follows. Even if the vehicle has a small deceleration while stopping, the acceleration suddenly disappears to zero because the relative motion with the ground disappears as soon as the vehicle stops. As a result, the magnitude of the acceleration of the vehicle is greatly reduced discontinuously, and it is represented by a very large jerk. The passenger suddenly feels the uncomfortable feeling of suddenly turning back due to the sudden change of acceleration. In this study, the magnitude of the suddenly changing acceleration is slowly decreased from immediately before stopping, so that the instantaneous acceleration of the vehicle can be converged to zero. If control is successful and successful behavior is achieved, it can be seen that acceleration and velocity converge smoothly to zero at the same time as shown in Fig. 2, and jerk is also considerably reduced in size
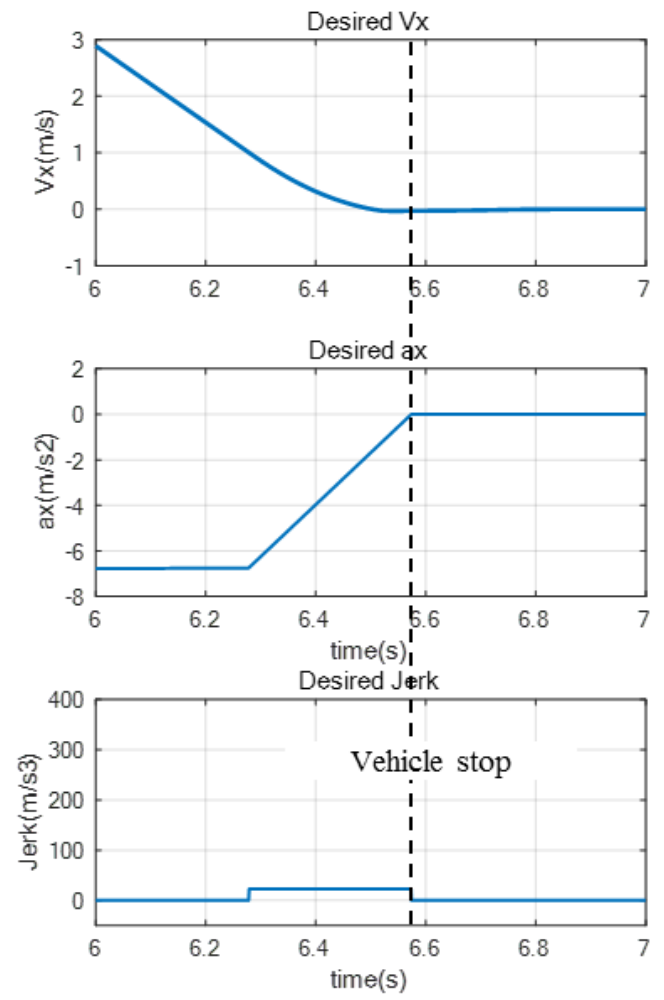

Figure 2. Desired vehicle speed, acceleration and jerk at vehicle stop for ride comfort

\section{Controller design}

The structure of the controller designed in this study is divided into the part that generates the desired acceleration trajectory for the control target and the controller that can track it well.

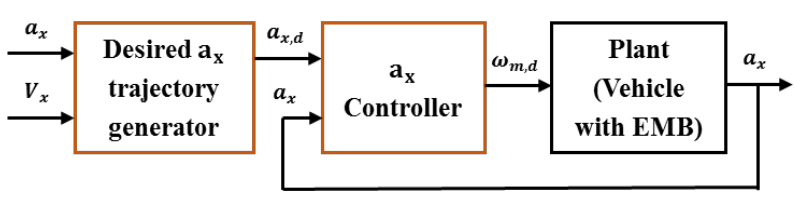

Figure 3. Control algorithm overview

\subsection{Desired acceleration trajectory generator}

The minimum jerk model proposed by Flash and Hogan for the objective evaluation of ride comfort has been studied to minimize this value by integrating the mean of the jerk squares as shown in Eq. (1) [3].

$$
C=\int_{t_{i}}^{t_{f}}\left(\frac{d^{3} x}{d t^{3}}\right)^{2} d t
$$

Where $d^{3} x / d t^{3}$ is longitudinal jerk, $t_{i}$ is initial time of the control, and $t_{f}$ is final time of the control. In order to minimize the discomfort index in the braking situation, the magnitude of acceleration at the moment when the 
vehicle is stopped should also converge to 0 at the same time in order to prevent the acceleration of the moment when the vehicle stops. In order to minimize the discomfort index under these conditions, it should be constant because the value obtained by integrating the jerk should be equal to the magnitude of the acceleration at the moment of initiation of control as shown in equation (3).

$$
\begin{gathered}
a_{x}\left(t_{f}\right)=0, v_{x}\left(t_{f}\right)=0 \\
\int_{t_{i}}^{t_{f}} \frac{d^{3} x}{d t^{3}} d t=a_{x}\left(t_{f}\right)-a_{x}\left(t_{i}\right)=-a_{x}\left(t_{i}\right) \\
j_{x, d}(t)=\frac{d^{3} x_{d}}{d t^{3}}=\text { const. } \\
a_{x}\left(t_{f}\right)=a_{x}\left(t_{i}\right)+j_{x, d} \cdot\left(t_{f}-t_{i}\right)=0 \\
v_{x}\left(t_{f}\right)=v_{x}\left(t_{i}\right)+a_{x}\left(t_{i}\right) \cdot\left(t_{f}-t_{i}\right)+\frac{1}{2} j_{x, d} \cdot\left(t_{f}-t_{i}\right)^{2}=0 \\
j_{x, d}=\frac{a_{x}\left(t_{i}\right)^{2}}{2 v_{x}\left(t_{i}\right)}
\end{gathered}
$$

Equations (4) and (5) express the condition that the speed and the acceleration become 0 at the same time when the vehicle stops. Through these equations, the desired jerk is expressed as (5), and the desired acceleration trajectory generator generates the desired acceleration trajectory through the acceleration and velocity information at the start of braking control.

$$
a_{x}(t)=a_{x}\left(t_{i}\right)+j_{x, d} \cdot\left(t-t_{i}\right)
$$

If the lower controller produces an ideal performance and follows exactly the designed acceleration trajectory, the discomfort index $\mathrm{C}$ is significantly improved as compared with the control without the control as shown in Table 1.

Table 1. Discomfort index when ideal braking control is performed

\begin{tabular}{|c|c|}
\hline & $\begin{array}{c}\text { Discomfort } \\
\text { index }\end{array}$ \\
\hline w/o control & 3254 \\
\hline $\begin{array}{c}\text { Ideal } \\
\text { control }\end{array}$ & 153 \\
\hline
\end{tabular}

\subsection{Plant modelling and acceleration controller}

In this study, braking control was performed through Electro Mechanical Brake system (EMB). It is a system that applies force to the brake through a motor, not a brake that moves the piston through the existing hydraulic pressure. A typical DC motor is used for the motor, and the dynamics inside the EMB are expressed simply as Equation 8-10 [4].

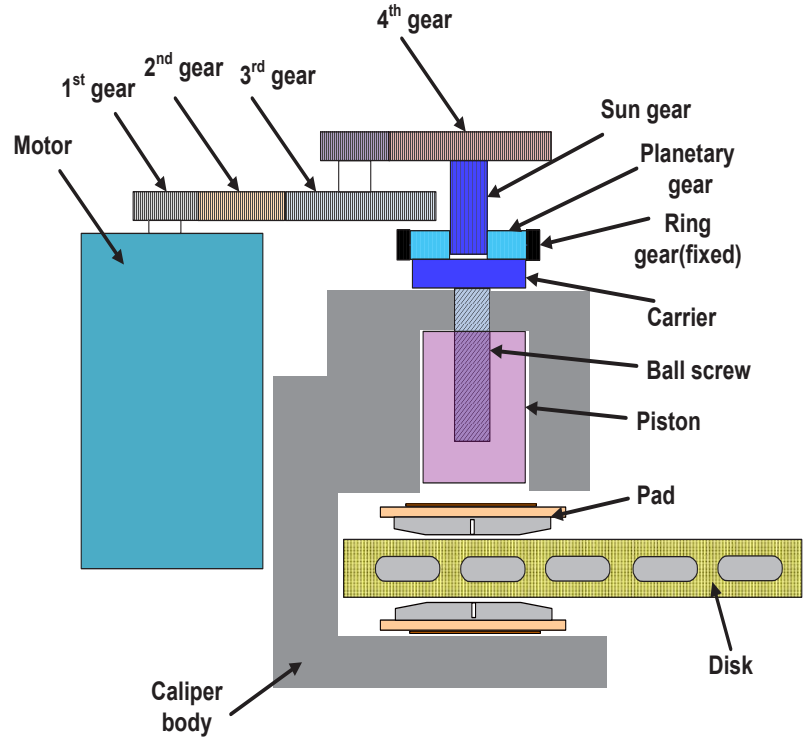

Figure 4. Electro mechanical brake system

$$
\begin{gathered}
T_{m}=k_{t} i_{q} \\
J \& \&=T_{m}-T_{f}-T_{L} \\
F_{c l}=k \theta
\end{gathered}
$$

To control the clamping force of the EMB with this dynamics, the desired motor speed is calculated through a longitudinal acceleration controller, which is implemented via PI type speed controller and current controller.

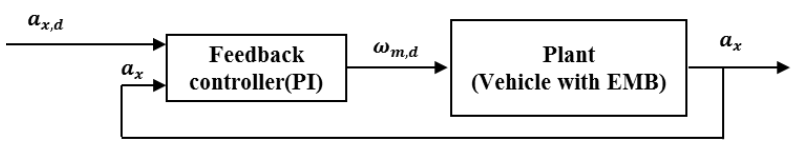

Figure 5. Internal control algorithm of EMB

Since the acceleration of the vehicle is proportional to the motor position of the EMB, the motor position control of the EMB is implemented through the PI controller to track the desired acceleration trajectory generated in Section 3.1.

\subsection{Trajectory update algorithm}

The target acceleration trajectory in this study should have zero longitudinal velocity and longitudinal acceleration simultaneously. Therefore, if perfect tracking is not achieved, it is difficult to achieve the existing goal. Although the EMB has a wide band width due to its speed in terms of reaction speed compared to conventional hydraulic brakes, control may fail even with a slight phase lag due to the controller and the plant. Therefore, it is necessary to design an algorithm that increases the control success rate by continuously updating the desired trajectory while controlling the desired trajectory rather than tracking it at a time when control starts. 
In this study, we assume that the sampling rate of the speed measured by GPS is $20 \mathrm{~Hz}$, and update the trajectory every $50 \mathrm{~ms}$ to implement tracking control.

\section{Simulation Results}

The simulation algorithm was implemented through Matlab Simulink and validated through a vehicle simulator, Carsim. The scenario started from the moment when the vehicle was at braking and the vehicle speed was $1 \mathrm{~m} / \mathrm{s}$.

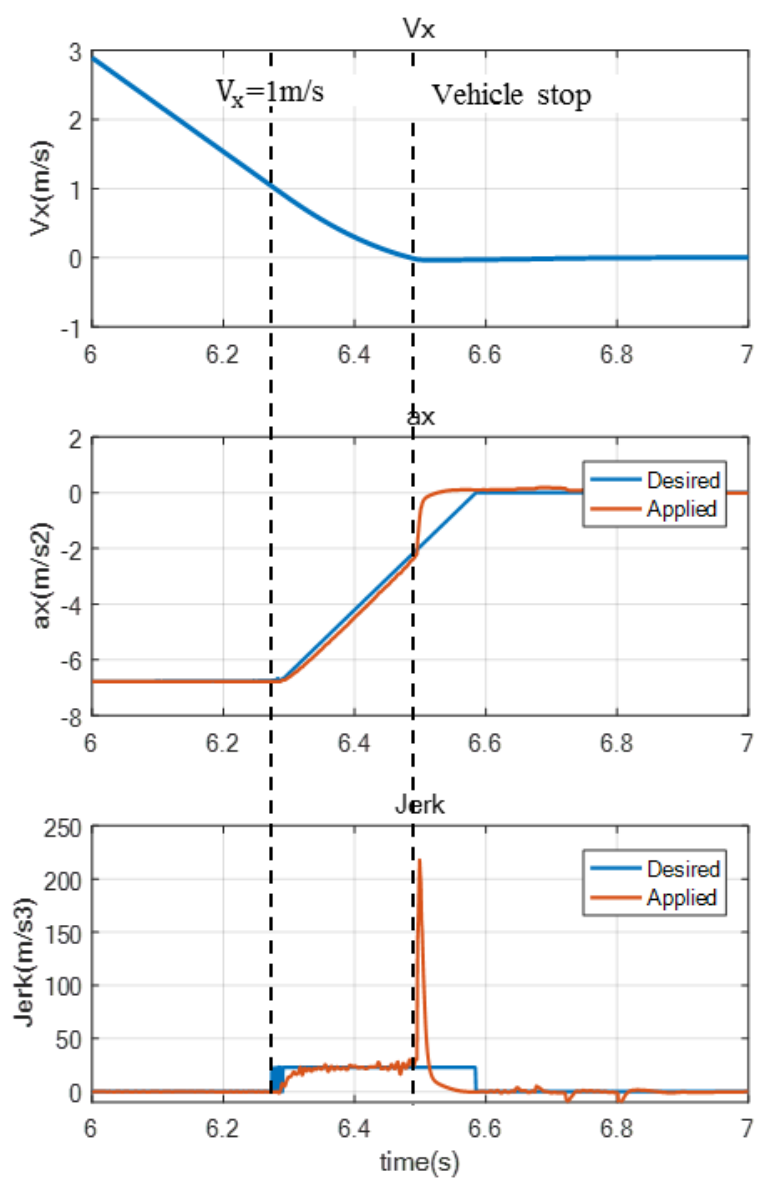

Figure 6. Control result without update algorithm

Figure 6 shows the result of tracking control after creating one desired trajectory. The jerk was decreased compared with the case where the control was not performed. However, as the phase lag caused the vehicle to stop, there was an acceleration, which resulted in a large jerk.

Figure 7 shows the result of simulation by adding the algorithm that updates the desired trajectory. When trajectory is updated every $50 \mathrm{~ms}$, it is confirmed that a desired trajectory is corrected to compensate for the error caused by the initial phase lag of the control. Thus, it is confirmed that the control finally succeeded.

The control performance for each algorithm is summarized in Table 2.

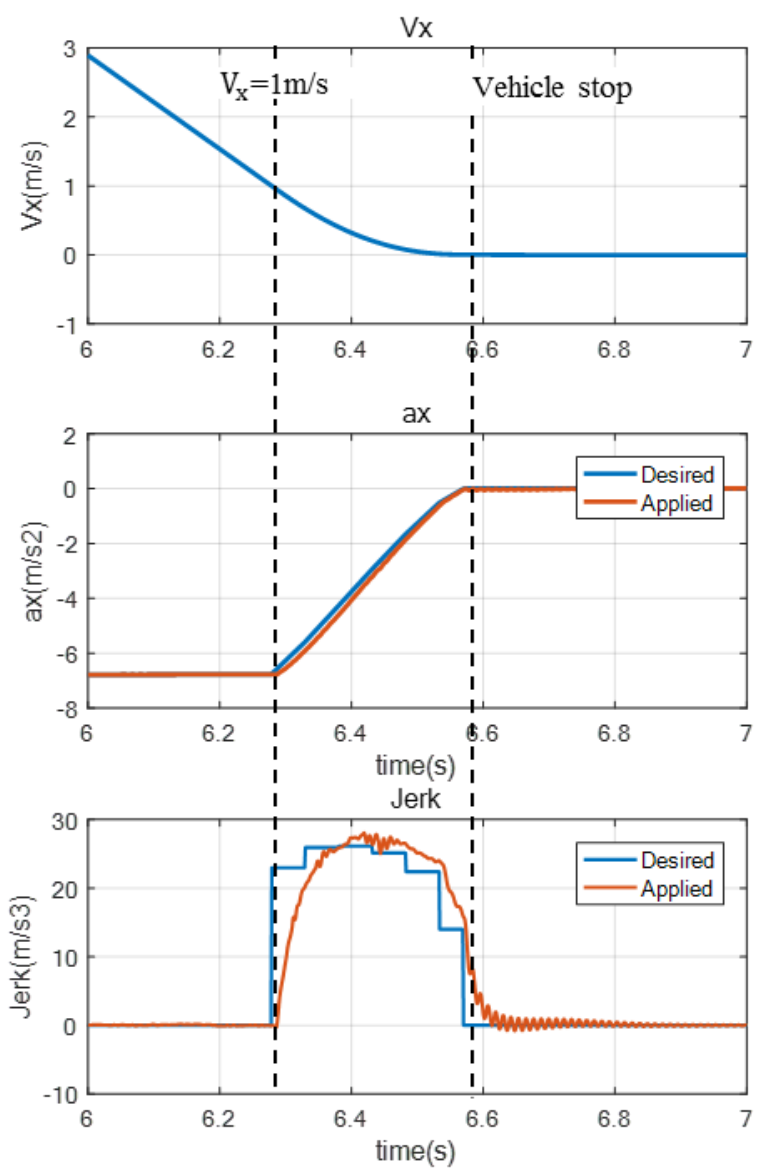

Figure 7. Control result with update algorithm

Table 2. Control performance comparison

\begin{tabular}{|c|c|c|}
\hline & $\begin{array}{c}\text { Discomfort } \\
\text { index }\end{array}$ & Max jerk \\
\hline w/o control & 3254 & 718 \\
\hline Ideal control & 153 & 23 \\
\hline $\begin{array}{c}\text { Without update } \\
\text { algorithm }\end{array}$ & 423 & 217 \\
\hline $\begin{array}{c}\text { With update } \\
\text { algorithm }\end{array}$ & 175 & 31 \\
\hline
\end{tabular}

The discomfort index is much smaller than without control when there is no update algorithm, but it is significantly lower than expected performance. When the trajectory update algorithm is added, the result is similar to the expected performance in the ideal control situation. The biggest side effect of braking control for ride comfort is the increase of braking distance. However, since the control starts at a low speed of $1 \mathrm{~m} / \mathrm{s}$ just before the vehicle stops, the braking distance increased by $2 \mathrm{~cm}$.

\section{Conclusion}


In order to control the passengers' ride comfort, we constructed an algorithm that reduces the brake input just before the vehicle stops. To do this, we created an acceleration trajectory that minimizes the discomfort index defined by the magnitude of jerk. We also designed PI controller based acceleration control algorithm and EMB internal control algorithm. We also added a trajectory update algorithm to solve the problem caused by phase lag between controller and plant. As a result, the results were significantly better than those without control. Although there was a great improvement in ride comfort, the increase in the braking distance, which is a side effect of this control, was very small, and the actual implementation feasibility of the algorithm is also sufficient.

\section{Acknowledgment}

This work was supported in parts by the National Research Foundation of Korea grant funded by the Korea government (MSIP) (No.2017R1A2B4004116), the Ministry of Science and ICT, Korea, under the Information Technology Research Center support program (IITP-2017-2012-0-00628) supervised by the Institute for Information and communications Technology Promotion, and the BK21 Plus Program.

\section{Reference}

1. H Seki, K Ishihara, S Tadakuma, " Novel Regenerative Braking Control of Electric PowerAssisted Wheelchair for Safety Downhill Road Driving", IEEE Transactions on Industrial Electronics, Volume: 56, Issue: 5, May 2009

2. Jun-Koo Kang and Seung-Ki Sul, "Vertical-vibration control of elevator using estimated car acceleration feedback compensation," in IEEE Transactions on Industrial Electronics, vol. 47, no. 1, pp. 91-99, Feb 2000
3. T Flash, N Hogan, "The coordination of arm movements: an experimentally confirmed mathematical model", Journal of Neuroscience, 1 July 1985, 5 (7) 1688-1703

4. C Line, C Manzie, MC Good, J Nell, P Rieth, "Electromechanical Brake Modeling and control", IEEE Transactions on Control Systems Technology, Volume: 16, Issue: 3, May 2008 